\title{
The Dynamics of Memory Consolidation of Landmarks
}

\author{
Janneke van Ekert (D), ${ }^{1,2 *}$ Joost Wegman, ${ }^{1,2}$ Clemens Jansen, ${ }^{1,2}$ \\ Atsuko Takashima, ${ }^{1,2,3}$ and Gabriele Janzen ${ }^{1,2}$
}

\begin{abstract}
Navigating through space is fundamental to human nature and requires the ability to retrieve relevant information from the remote past. With the passage of time, some memories become generic, capturing only a sense of familiarity. Yet, others maintain precision, even when acquired decades ago. Understanding the dynamics of memory consolidation is a major challenge to neuroscientists. Using functional magnetic resonance imaging, we systematically examined the effects of time and spatial context on the neural representation of landmark recognition memory. An equal number of male and female subjects (males $N=10$, total $N=20$ ) watched a route through a large-scale virtual environment. Landmarks occurred at navigationally relevant and irrelevant locations along the route. Recognition memory for landmarks was tested directly following encoding, $24 \mathrm{~h}$ later and 30 days later. Surprisingly, changes over time in the neural representation of navigationally relevant landmarks differed between males and females. In males, relevant landmarks selectively engaged the parahippocampal gyrus (PHG) regardless of the age of the memory. In females, the response to relevant landmarks gradually diminished with time in the PHG but strengthened progressively in the inferior frontal gyrus (IFG). Based on what is known about the functioning of the PHG and IFG, the findings of this study suggest that males maintain access to the initially formed spatial representation of landmarks whereas females become strongly dependent on a verbal representation of landmarks with time. Our findings yield a clear objective for future studies. (c) 2016 Wiley Periodicals, Inc.
\end{abstract}

KEY WORDS: $\quad$ spatial memory; navigation; sex differences; parahippocampal gyrus; inferior frontal gyrus

\section{INTRODUCTION}

Navigating through space is fundamental to human nature and requires the ability to recollect memories from the recent and remote past (Burgess et al., 2002; Spiers and Maguire, 2007; Squire et al.,

\footnotetext{
${ }^{1}$ Radboud University Nijmegen Behavioural Science Institute, P.O. Box 9104, $6500 \mathrm{HE}$, Nijmegen, The Netherlands; ${ }^{2}$ Radboud University Nijmegen, Donders Institute for Brain, Cognition and Behaviour, P.O. Box 9101, $6500 \mathrm{HB}$, Nijmegen, The Netherlands; ${ }^{3}$ Max Planck Institute for Psycholinguistics, P.O. Box 310, 6500 AH, Nijmegen, The Netherlands Grant sponsor: Nederlandse Organisatie voor Wetenschappelijk Onderzoek (NWO); Vidi; Grant number: 452-07-015; Grant sponsor: European Research Council (ERC); ERC Starting Grant; Grant number: 204643.

*Correspondence to: Janneke van Ekert, Radboud University Nijmegen, Behavioural Science Institute, P.O. Box 9104, 6500 HE Nijmegen, The Netherlands. E-mail: j.vanekert@pwo.ru.nl

Accepted for publication 19 December 2016.

DOI 10.1002/hipo.22698

Published online 29 December 2016 in Wiley Online Library (wileyonlinelibrary.com).
}

2015). Neuroscientists have emphasized the importance of the medial temporal lobe, including the hippocampus and the parahippocampal gyrus (PHG), in recent and remote spatial memory (O'Keefe and Nadel, 1978; Burgess et al., 2002; Shelton and Gabrieli, 2002; Hassabis et al., 2009; Baumann et al., 2010). It has been suggested that the hippocampus and the posterior PHG are crucial for the retrieval of memories as long as they entail contextual cues (Diana et al., 2007; Eichenbaum et al., 2007). Here, we explicitly investigate the neural mechanism underlying memory consolidation of landmarks and their associated spatial context over the course of 1 month.

Functional brain imaging studies have delineated a role for the posterior PHG in tasks requiring spatial processing, including (but not exclusively) scene perception (Epstein and Kanwisher, 1998; Linsley and MacEvoy, 2015), topographical learning (Aguirre et al., 1996; Maguire et al., 1998), memory for object-location associations (Duzel et al., 2003; Hayes et al., 2004; Sommer et al., 2005), and memory for landmarks (Janzen and van Turennout, 2004; Rosenbaum et al., 2004; for a review on the involvement of the PHG in spatial processing, see Maguire et al., 1999). In recent years, attempts have been made to reconcile these findings in a single explanatory model on the role of the posterior PHG. Specifically, it has been suggested that the posterior PHG supports representations of the spatio-temporal context associated with objects that are being processed (Diana et al., 2007; Eichenbaum et al., 2007; Ranganath and Ritchey, 2012; Aminoff et al., 2013).

A series of studies on landmark recognition memory support the theory that the posterior PHG is crucial for contextual associations (Janzen and van Turennout, 2004; Janzen and Jansen, 2010; Schinazi and Epstein, 2010; Wegman and Janzen, 2011). In those studies, the location at which landmarks occurred was systematically manipulated to test the hypothesis that memory for landmarks varies as a function of the associated spatial context. Results indicated that landmarks encountered at a navigationally relevant location (i.e., an intersection, referred to as a decision point; DP) engaged the posterior PHG during encoding and subsequent retrieval. No such a response was observed for landmarks encountered at an irrelevant location (i.e., a simple turn, referred to as non-decision point; NDP). Importantly, this effect was maintained over a period of $24 \mathrm{~h}$ (Janzen et al., 2007, 2008). 
The posterior PHG is considered one of the major neocortical inputs to the hippocampus. This anatomical connection is illustrative of a close functional relationship between the two regions. Whereas the posterior PHG encodes the spatiotemporal context of an object that is to be remembered, the hippocampus integrates object information and contextual information into a coherent memory representation (Goh et al., 2004; Howard et al., 2011). Whether the role of the posterior PHG and the hippocampus is time limited is still subject of debate (Hirshhorn et al., 2012; Boccia et al., 2014).

Traditionally, the role of the hippocampus is thought to gradually decline when memories age. As consolidation proceeds, stored information becomes reorganized in a distributed neocortical network that mediates retrieval independently of the hippocampus (McClelland et al., 1995; Squire and Alvarez, 1995). The occurrence of this process, conceptualized as the standard theory of consolidation, is supported by a substantial body of experimental studies in nonhuman primates and rodents (Winocur, 1990; Zola-Morgan and Squire, 1990; Kim and Fanselow, 1992; Bontempi et al., 1999; Maviel et al., 2004) and neuroimaging studies in humans (Haist et al., 2001; Douville et al., 2005; Takashima et al., 2006, 2009; Smith and Squire, 2009; Yamashita et al., 2009). Collectively, these studies outline a critical role for the hippocampus in recent but not remote memory. On the contrary, neocortical areas including the posterior PHG and the prefrontal cortex have been linked to remote memory (Wiltgen et al., 2004; Frankland and Bontempi, 2005; Takashima et al., 2006; Nieuwenhuis and Takashima, 2011; Euston et al., 2012). Specifically, Remondes and Schuman (2004) showed that disrupting entorhinal projections to the hippocampus in rats prevents recently acquired spatial memories from becoming consolidated in the neocortex. This finding suggests that areas with strong anatomical connections to the entorhinal cortex, for example the posterior PHG, play a crucial role in remote memory.

Alternative theories (multiple trace theory (MTT), binding of item and context (BIC) model, scene construction theory, transformation hypothesis) instead propose that the hippocampus is crucial for the retrieval of both recent and remote memories for as long as they retain a great level of detail (Nadel and Moscovitch, 1997; Eichenbaum et al., 2007; Hassabis and Maguire, 2007; Winocur et al., 2010). While all theories recognize the vital role of the hippocampus, the BIC model is the only one that highlights the importance of the posterior PHG in the retrieval of detailed remote memories (Diana et al., 2007; Eichenbaum et al., 2007). According to the BIC model, there is dynamic interplay between the hippocampus and the posterior PHG, such that hippocampal activity during retrieval will reactivate contextual associations in the posterior PHG. As a result, detailed memory - of which contextual associations are the principal element - produce activation in both the hippocampus and the posterior PHG irrespective of the age of the memory. In line with the BIC model, Burwell et al. (2004) demonstrated that the equivalent of the posterior PHG in rats is involved in the storage, maintenance and retrieval of memory for contextual details for a period of at least 100 days.
Moreover, studies on patients with damage to the hippocampus and the posterior PHG have documented impaired memory for topographic details such as landmarks and their spatial context that make for a rich representation of the environment (Rosenbaum et al., 2000; Herdman et al., 2015). Together, those studies postulate a permanent role for the PHG in contextual memory.

According to those alternative theories, however, memories can also be recalled without reference to the context in which they have been formed. Those memories are semanticized rather than contextually detailed and a considerable amount of literature has been published on the process of semanticization (see e.g., Moscovitch et al., 2005). Research into the representation of semanticized memories points to a distributed neocortical network (Cabeza and Nyberg, 2000; Thompson-Schill, 2003; Binder and Desai, 2011). Specifically, evidence suggests that the inferior frontal gyrus (IFG) is implicated in verbally mediated associative memory (Takashima et al., 2009, in press). This implies that the IFG may be involved in semanticized memory for landmarks.

The aim of this study is to examine the effects of time and spatial context. An equal number of male and female participants watched a video depicting a route through a large-scale virtual environment (Fig. 1a). They were instructed to remember the route and the objects along the route. The location at which objects occurred was manipulated to test the hypothesis that the process of memory consolidation varies as a function of the associated spatial context. To that end, half of the objects occurred at a navigationally relevant location (i.e., a decision point; DP) and half of the objects occurred at an irrelevant location (i.e., a non-decision point; NDP) along the route. Directly after the study phase (day 0 ), participants performed an old-new recognition memory test on a subset of the objects (Fig. 1b). Recognition memory for different subsets was tested $24 \mathrm{~h}$ (day 1) and 30 days (day 30) later. During all three memory tests, brain activity was measured using functional magnetic resonance imaging (fMRI).

In line with previous research, we expected enhanced PHG activation when landmarks are associated with a relevant spatial context compared with landmarks associated with an irrelevant spatial context. Though the standard theory of consolidation is not explicit about the role of the posterior PHG in recent and remote memory, it can be derived from previous studies that neocortical areas like the posterior PHG are involved in both recent and remote memory. Alternative theories such as the BIC model likewise predict a role for the posterior PHG in recent and remote memory, but only when the dominant memory is accompanied by an association with the spatial context.

In addition, exploratory analysis was conducted aiming to evaluate the role of sex in memory for landmarks. There is a growing body of literature that recognizes the effect of sex on spatial memory (Grön et al., 2000; Andreano and Cahill, 2009). Behavioral studies indicate that men make use of geometric information of space whereas women are more adept at using landmark information to guide successful navigation 
a
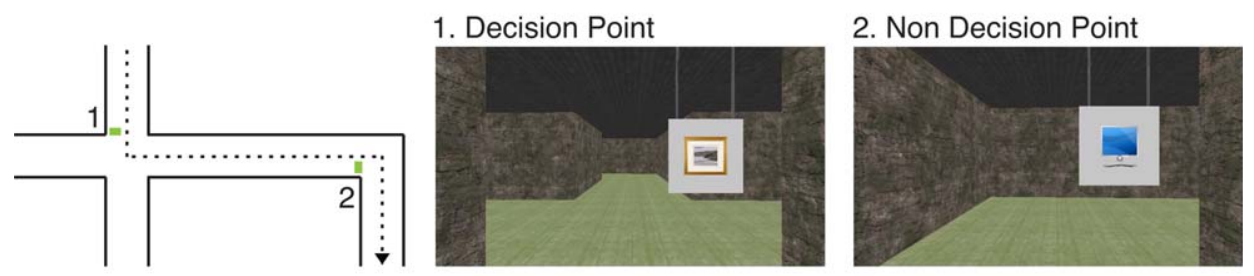

b
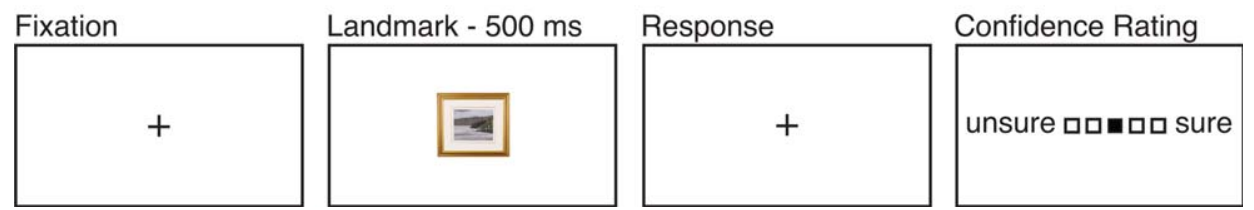

FIGURE 1. Experimental Design. (a) Subjects watched a film sequence through a large-scale virtual environment. They were instructed to remember the route and the objects along the route. Half of the objects occurred at DPs and half of the objects occurred at NDPs. (b) Subjects performed an old-new recognition task on $1 / 3$ of the objects directly after the study phase (day 0), 24 h later (day 1), and 30 days later (day 30). During all three memory tests, brain activation patterns were measured using fMRI. [Color figure can be viewed at wileyonlinelibrary.com]

(Lawton, 1994; Maguire et al., 1999; Montello et al., 1999; Saucier et al., 2002; Holden et al., 2015). Lesion studies in rodents (Roof et al., 1993; Kolb and Cioe, 1996) and imaging studies in humans (Grön et al., 2000; Sneider et al., 2011; Persson et al., 2013) suggest that the tendency for men and women to use different strategies may be mediated by distinct anatomy. While males predominantly rely on the hippocampus, females activate the prefrontal cortex. In light of these findings, it has become extremely difficult to ignore the role of sex in spatial cognition. This has led to a proliferation of studies that added sex to the statistical model (Janzen and van Turennout, 2004; Janzen et al., 2007). Here, we report one such study.

\section{MATERIALS AND METHODS}

\section{Participants}

A group of 22 adult human participants (11 females) were recruited from the Radboud University Nijmegen community. They provided informed consent according to the Declaration of Helsinki prior to participating in the study. Data from two participants ( 1 female) were discarded from analyses due to the presence of anomalies in the brain. As such, 20 participants contributed to the final sample. Participants were right-handed according to self-report, had normal or corrected-to-normal vision and did not have a history of neurological impairment. Ages ranged from 19 to 27 years $(M=22.48, S D=2.50)$. A two-sample $t$-test confirmed that distribution of age was equal in male and female groups $(t(18)=-0.71, P=0.489)$. All participants received monetary reward or course credits for participation. The study was approved by the CMO committee on Research Involving Human Participants (Region ArnhemNijmegen).

\section{Materials and Procedure}

The study took place on three separate days; day 0 , day 1 , and day 30. Day 0 was divided into two parts: A behavioral study phase and a recognition memory task during which functional images of the brain were acquired. Following the functional run, we acquired a high resolution T1-weighted anatomical scan. On day 1 , a second recognition memory test was administered while fMRI time series were acquired. Thereafter, we collected diffusion-weighted data. On day 30, a third recognition memory test was administered during which functional images of the brain were acquired.

During the study phase on day 0, participants watched a film sequence of a tour through a virtual environment. They received the following standardized written instruction: "You applied for a job in a museum that exhibits belongings of famous people. You will be guided through four sections of the museum. After training, you should be able to guide a tour. Therefore, while you are watching the film, please pay attention to the route and the objects along the route." To ensure adequate encoding, participants watched the film sequence twice.

The virtual environment from which the film sequences were recorded was created using Unreal Tournament 3 (Epic Games). The environment had a maze-like layout, consisting of straight corridors alternated with intersections (decision point; DP) and simple turns (non-decision point; NDP). Corridors were $4.5 \mathrm{~m}$ wide and $3.4 \mathrm{~m}$ high. The length of the corridors was on average $6.7 \mathrm{~m}$, jittered between 5.6 and $7.9 \mathrm{~m}$ in steps of $0.28 \mathrm{~m}$. The viewpoint moved through the environment at a simulated eye-level of $1.70 \mathrm{~m}$, at a constant speed of 
$1.12 \mathrm{~m} / \mathrm{s}$. We selected 216 color photographs of common objects. Those photographs appeared on white posters along the route on either DP or NDP locations. As such, 108 DPs and 108 NDPs were marked with unique objects, each indicating a right or left turn (Fig. 1a). In the film sequences, each object remained visible for $3 \mathrm{~s}$ on average. Objects presented at DPs and NDPs were matched in terms of visual complexity and word frequency. In total, there were 4 film sequences shown from first person perspective, lasting about $7 \mathrm{~min}$ each. The presentation order of the 4 film sequences was counterbalanced over participants.

Next, participants performed a recognition memory task inside the scanner. We selected an additional 162 color photographs of common objects. These objects served as distracters and were matched to DP and NDP objects in terms of visual complexity and word frequency. The total number of objects was divided into three subsets (36 DP objects, 36 NDP objects, 54 distracters each). Recognition memory was tested on day 0 , day 1 , and day 30 . The order in which subsets were tested was counterbalanced over participants.

Each trial of the recognition memory test consisted of a fixation cross, followed by an object shown from canonical perspective on a white background for $500 \mathrm{~ms}$. Thus, during scanning, no context-related information was presented. All stimuli were presented in a randomly intermixed order to prevent participants from anticipating and changing strategies for the different event types. By button presses, participants indicated as quickly and as accurately as possible whether the object had occurred in the former film sequences. Participants subsequently rated their confidence on a 5-point Likert scale, 1 indicating they were very unsure and 5 indicating they were very sure. The average inter-stimulus interval was $4,000 \mathrm{~ms}$, jittered between $3,000 \mathrm{~ms}$ and $5,000 \mathrm{~ms}$ in steps of $250 \mathrm{~ms}$, counterbalanced over conditions (Fig. 1b).

\section{Image Acquisition}

Functional images of the whole brain were acquired on a 3T Siemens Trio scanner (Siemens, Erlangen, Germany). We used a multi gradient-echo planar scanning sequence to collect 36 axial slices covering the entire brain (voxel size $3.3 \mathrm{~mm} \times 3.3 \mathrm{~mm} \times 3 \mathrm{~mm}$, $\mathrm{TR}=2,390 \mathrm{~ms}, \mathrm{TE}=9.4 \mathrm{~ms}, 21.2 \mathrm{~ms}, 33 \mathrm{~ms}, 45 \mathrm{~ms}$, field of view $=212 \mathrm{~mm}$, flip angle $=90^{\circ}$ ). Following acquisition of functional images on day 0 , we acquired a high resolution $\mathrm{T} 1$ weighted anatomical scan (MP-RAGE; 192 sagittal slices, TR = 2,300 ms; $\mathrm{TE}=3.03 \mathrm{~ms} ; 8^{\circ}$ flip angle; slice thickness $=1 \mathrm{~mm}$; $\mathrm{FOV}=256 \mathrm{~mm}$; GRAPPA parallel imaging with an acceleration factor of 2). After administration of the recognition memory test on day 1 , we additionally collected diffusion-weighted data which are published elsewhere (Wegman et al., 2014).

\section{Image Processing and Data Analysis}

fMRI data were preprocessed and analyzed with SPM8 (www.fil.ion.ucl.ac.uk/spm). All volumes were realigned to the first echo of the first volume. To combine multiple echoes for each volume, we then calculated echo weights based on the first
30 volumes and applied those weights to the time series (Poser et al., 2006). The functional images were slice time corrected, and the subject mean was coregistered with the corresponding T1-weighted structural scan using normalized mutual information optimization. The structural image was segmented into grey matter, white matter and cerebrospinal fluid, functional images were spatially normalized and transformed into common space, as defined by the Montreal Neurological Institute (MNI) T1 template. Finally, the images were spatially filtered by convolving them with an isotropic 3D Gaussian kernel (6 $\mathrm{mm}$ full width at half maximum).

Statistical analyses were performed within the framework of the GLM. On the single subject level, we contrasted each condition of interest (DP and NDP objects that were adequately remembered and rated as $2-5$ on a 5 level confidence scale on day 0 , day 1 , and day 30) with a low level visual baseline (fixation cross). The resulting contrast images were entered into a full factorial second-level analysis with one between subject factor (Sex, Male/Female) and two within subject-factors (Context, DP/NDP; Time, Day0/Day1/Day30). A total of three covariates were included in the model to control for behavioral differences in recognition memory performance, response time and confidence rating. The results for the analyses were initially thresholded at voxel level $P=0.001$ (uncorrected) and the cluster-size statistics were used as the test statistic (Hayasaka and Nichols, 2003). Only clusters at $P \leq 0.05$ (family-wise error corrected: FWE) were considered significant. Given the role of the medial temporal lobe as the primary region of interest, region specific investigations were performed by using anatomical masks of the bilateral posterior PHG (equal split of the PHG into anterior PHG and posterior PHG) and the bilateral hippocampus using the Anatomical Automatic Labeling atlas (Maldjian et al., 2003). The results of those analyses were initially thresholded at voxel level $P=0.001$ (uncorrected), and small volume correction was applied on the clusterlevel for multiple comparisons $\left(P_{\mathrm{SVC}}<0.05\right)$. All local maxima are reported in MNI coordinates.

\section{RESULTS}

\section{Behavioral Results}

Behavioral data indicated that recognition memory for objects (probability of a hit minus probability of a false alarm, where $0 \%$ represents chance level) remained significantly above chance on all three occasions (day 0: $M=80.15 \%$, $t(19)=22.29, P<0.001$; day $1: M=62.81 \%, t(19)=15.90$, $P<0.001$; day 30: $M=18.35 \%, t(19)=7.13, P<0.001)$. A mixed analysis of variance with the factors Time (day 0 , day 1 , day 3), Context (DP, NDP) and Sex (males, females, see Table 1) showed that memory performance decreased significantly with time $(F(2,36)=201.87, P<0.001)$, both from day 0 to day $1(F(1,18)=103.91, P<0.001)$ and from day 1 to day $30(F(1,18)=159.32, P<0.001)$. Neither the Time*Context 
TABLE 1.

Summary of Behavioral Measures

\begin{tabular}{|c|c|c|c|c|c|c|c|}
\hline & & \multicolumn{3}{|c|}{ Males } & \multicolumn{3}{|c|}{ Females } \\
\hline & & Day 0 & Day 1 & Day 30 & Day 0 & Day 1 & Day 30 \\
\hline \multirow[t]{4}{*}{$P($ hit $)-P(f a)$} & $\mathrm{DP}$ & 80.82 & 65.13 & 22.97 & 83.23 & 61.53 & 12.19 \\
\hline & & 5.21 & 6.05 & 4.21 & 5.21 & 6.05 & 4.21 \\
\hline & NDP & 80.25 & 59.41 & 21.54 & 76.29 & 65.19 & 16.70 \\
\hline & & 5.57 & 5.95 & 3.84 & 5.57 & 5.95 & 3.84 \\
\hline \multirow[t]{4}{*}{ RT } & $\mathrm{DP}$ & 972.08 & 998.94 & 1525.12 & 1036.85 & 1031.91 & 1371.08 \\
\hline & & 66.01 & 54.16 & 130.89 & 66.01 & 54.19 & 130.89 \\
\hline & NDP & 988.77 & 1078.01 & 1552.35 & 1030.21 & 1001.65 & 1306.28 \\
\hline & & 65.72 & 68.91 & 116.37 & 65.72 & 68.91 & 116.37 \\
\hline
\end{tabular}

Note. Means and standard errors of the means are presented in the table.

$\mathrm{P}($ hit): probability of a hit, $\mathrm{P}(\mathrm{fa})$ : probability of a false alarm, RT: response time in milliseconds.

interaction effect nor the Time* ${ }^{*}$ ex interaction effect reached statistical significance $\quad($ Time*Context, $F(2, \quad 36)=1.44$, $P=0.250$; Time* ${ }^{*}$ ex $\left., F(2,36)=1.09, P=0.346\right)$. However, the data showed a significant Time $^{*}$ Context $^{*}$ Sex interaction effect $(F(2,36)=3.52, P=0.040)$, indicating a lower rate of forgetting for DP objects compared with NDP objects in males, whereas the reverse pattern was evident for females. Post hoc analyses demonstrated that this interaction was significant from day 0 to day $1(F(1,18)=9.33, P=0.007)$, but not from day 1 to day $30(F(1,18)=0.27, P=0.607)$.

In addition, the time needed to respond to objects presented in the recognition memory test was analyzed in a mixed analysis of variance with the factors Time (day 0 , day 1 , day 3 ), Context (DP, NDP) and Sex (males, females, see Table 1). Like memory performance, response times changed significantly with time $(F(2,36)=29.08, P<0.001)$. Post hoc analyses did not reveal significant changes from day 0 to day $1(F(1,18)=0.25$, $P=0.624)$, however they showed a significant increase between day 1 and day $30(F(1,18)=35.97, P<0.001)$. The increase in response times did not differ between DP and NDP objects (Time*Context: $F(2,36)=0.52, P=0.598)$ or between males and females (Time*Sex: $F(2,36)=2.30, P=0.115)$. Moreover, the data did not demonstrate a Time ${ }^{*}$ Context $^{*}$ Sex interaction effect $(F(2,36)=0.24, P=0.791)$. Importantly, the results demonstrated a significant Context ${ }^{*}$ Sex interaction effect $(F(1,18)=12.02, P=0.003)$, suggesting that in general male participants responded faster to DP objects compared with NDP objects while female participants responded faster to NDP objects compared with DP objects.

\section{fMRI Results}

\section{The effect of time on memory for landmarks}

The neural correlates of landmark recognition memory were investigated within the framework of the general linear model (GLM). First, the effect of Time on landmark recognition memory was considered. The data revealed a significant decrease in activation from day 0 to day 1 in the left hippocampus $\left(x=-24 \quad y=-24 \quad z=-12, P_{\mathrm{SVC}}=0.013\right)$, as well as in other regions of the medial system (Table 2) that have frequently been associated with memory (Ranganath and Ritchey, 2012). No significant decreases were observed from day 1 to day 30. Furthermore, the data did not reveal neural increases from day 0 to day 1 or day 1 to day 30 .

\section{The effect of time on memory for landmarks in the male and female brain}

To dissociate the contribution of both sexes, the effect of Time on landmark recognition memory was evaluated separately for males and females. A decrease in activation was observed in females from day 0 to day 1 in the left hippocampus $(x=-22$ $\left.y=-26 z=-8, P_{\mathrm{SVC}}=0.007\right)$. By contrast, this effect was not observed in males. This pattern of results should be interpreted with caution because the interaction effect in that region was not significant (Time* ${ }^{*}$ ex: $x=-22 \quad y=-28 \quad z=-6$, $\left.P_{\mathrm{SVC}}=0.099\right)$. Moreover, decreases in retrieval-related activity were observed in other regions of the female brain (Table 3). These results suggest that the general decreases reported in Table 2 were driven by females, although the Time*Sex interaction effects in those regions did not reach statistical significance.

No significant increases with time were observed in either males or females. However, a significant Time* Sex interaction effect in the left PHG $(x=-28 \quad y=-38 \quad z=-6$, $\left.P_{\text {SVC }}=0.043\right)$ suggested an increase in retrieval-related activation from day 1 to day 30 in males whereas activity remained stable in females during that time period.

\section{The effect of spatial context on recent and remote memory landmarks}

To examine differences in the neural response to landmarks between those associated with a relevant spatial context and those associated with an irrelevant spatial context, DP landmarks were contrasted with NDP landmarks separately for day 0 , day 1 , and day 30 . While the data failed to reveal a selective response to DP landmarks on day 0 , a significant effect was 
TABLE 2.

Clusters of Activation for the Contrast Day $0>$ Day 1

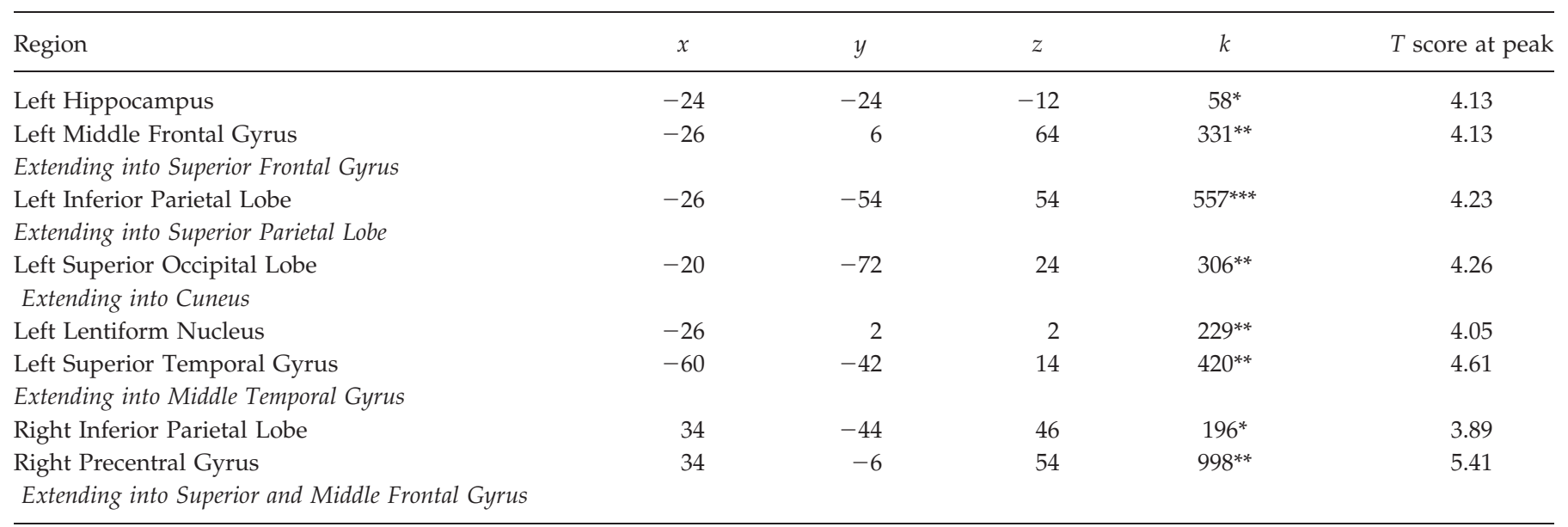

Note. The $x, y, z$ coordinates of local maxima are displayed in MNI standard space coordinates. Whole brain threshold $P<0.001$, uncorrected. $k=$ cluster size. ${ }^{*} P<0.05$ at cluster level, ${ }^{* *} P<0.01$ at cluster level, ${ }^{* * *} P<0.001$ at cluster level.

observed on day 1 in the right posterior PHG $(x=18 y=-26$ $\left.z=-18, k=22, P_{\mathrm{SVC}}=0.035\right)$ and in the right thalamus $\left(x=16 y=-10 z=14, k=176, P_{\mathrm{FWE}}=0.022\right)$. Moreover, on day 30 a selective response to DP landmarks was associated with increased activity in the right IFG $(x=28 y=12$ $\left.z=-16, k=261, P_{\mathrm{FWE}}=0.003\right)$ and, although not significant, in the left IFG $(x=-42 y=14 z=-16, k=112$, $\left.P_{\mathrm{FWE}}=0.115\right)$. The findings suggest that the selective response to DP landmarks gradually diminished with time in the PHG but strengthened progressively in the IFG. It should be mentioned, however, that no Time*Context interaction effects were observed in those regions.
The data did reveal significant Time*Context interactions in other areas of the medial system (Table 4). These interactions were not indicative of gradual decreases or increases in retrieval-related activity for DP landmarks. Rather, the effects were driven by an increase in retrieval-related activity for NDP landmarks from day 1 to day 30 .

\section{The effect of spatial context on recent and remote memory for landmarks in males and females}

In addition, the effect of sex on the selective response to DP landmarks was evaluated on day 0 , day 1 , and day 30 . The data did not reveal significant Context*Sex interaction effects

TABLE 3.

Clusters of Activation for the Contrast Day 0 > Day 1 Separately for Males and Females

\begin{tabular}{|c|c|c|c|c|c|}
\hline Region & $x$ & $y$ & $z$ & $k$ & $T$ score at peak \\
\hline \multicolumn{6}{|l|}{ FEMALES } \\
\hline$\overline{\text { Left Hippocampus }}$ & -22 & -26 & -8 & $75^{* *}$ & 4.62 \\
\hline \multicolumn{6}{|c|}{ Extending into Superior Frontal Gyrus } \\
\hline Left Inferior Parietal Lobe & -28 & -46 & 56 & $253^{* *}$ & 4.50 \\
\hline \multicolumn{6}{|c|}{ Extending into Superior Parietal Lobe } \\
\hline Left Lentiform Nucleus & -24 & 2 & 2 & $162^{*}$ & 4.11 \\
\hline Left Cingulate Gyrus & -8 & -14 & 28 & $263^{* *}$ & 5.29 \\
\hline Right Inferior Parietal Lobe & 34 & -46 & 46 & $289^{* *}$ & 4.48 \\
\hline Right Superior Frontal Gyrus & 26 & -4 & 62 & $1223^{* * *}$ & 5.23 \\
\hline \multicolumn{6}{|l|}{ Extending into Precentral Gyrus } \\
\hline
\end{tabular}

Note. The $x, y, z$ coordinates of local maxima are displayed in MNI standard space coordinates. Whole brain threshold $P<0.001$, uncorrected. $k=$ cluster size. ${ }^{*} P<0.05$ at cluster level, ${ }^{* *} P<0.01$ at cluster level, ${ }^{* * *} P<0.001$ at cluster level. 
TABLE 4.

Clusters of Activation for the Contrast Day 1(DP>NDP $)>$ Day 30(DP $>N D P)$

\begin{tabular}{|c|c|c|c|c|c|}
\hline Region & $x$ & $y$ & $z$ & $k$ & $T$ score at peak \\
\hline $\begin{array}{l}\text { Left Superior Temporal Lobe } \\
\text { Extending into Operculum and Insula }\end{array}$ & -38 & -28 & 2 & $549^{* * *}$ & 5.29 \\
\hline Left Middle Temporal Gyrus & -50 & -58 & 8 & $219^{* *}$ & 4.07 \\
\hline $\begin{array}{l}\text { Right Frontal Lobe } \\
\text { Extending into Anterior Cingulate }\end{array}$ & 22 & 42 & 10 & $577^{* * *}$ & 4.76 \\
\hline
\end{tabular}

Note. The $x, y, z$ coordinates of local maxima are displayed in MNI standard space coordinates. Whole brain threshold $P<0.001$, uncorrected. $k=$ cluster size. ${ }^{*} P<0.05$ at cluster level, ${ }^{* *} P<0.01$ at cluster level, ${ }^{* *} P<0.001$ at cluster level.

on day 0 or day 1 suggesting that initially, males and females recruit the right posterior PHG and the right thalamus equally. However, a significant Context*Sex interaction effect was

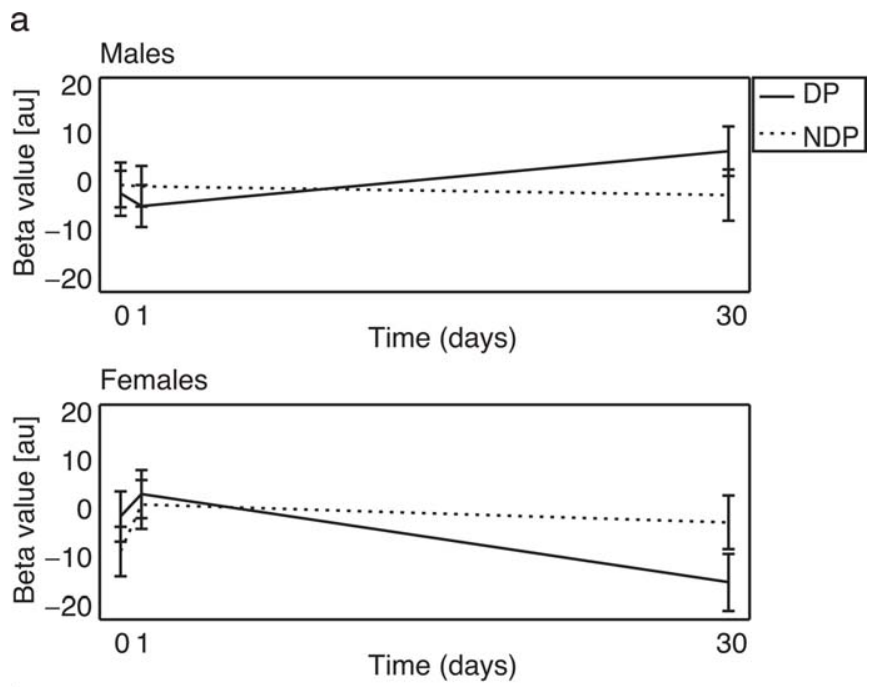

b

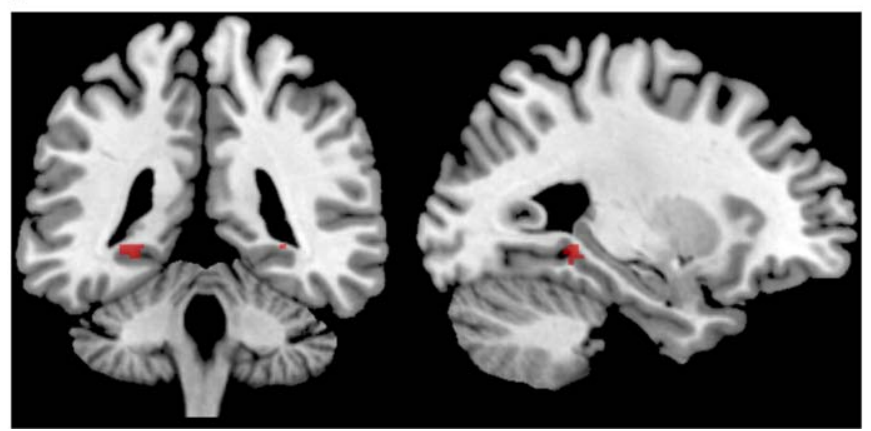

FIGURE 2. The process of memory consolidation varies as a function of context and sex. (a) The representation of DP $>$ NDP increased with time in the left PHG in males, whereas a decrease was observed in females. Note that the beta weights were extracted from the peak voxel $(x=-32 y=-42 z=-4, P=0.048)$. Weights were plotted for descriptive purposes only (b) Left PHG $(x=-32 y=-42 z=-4$, $P=0.048$ ). [Color figure can be viewed at wileyonlinelibrary.com] observed on day 30 in a large network within the medial temporal lobe $\left(x=-38 y=-42 z=8, k=242, P_{\mathrm{FWE}}=0.005\right)$ including the left PHG $(x=-28 y=-40 z=-6, k=43$, $\left.P_{\text {SVC }}=0.014\right)$. A selective response to DP landmarks in the PHG was evident in males. By contrast, the reverse pattern was
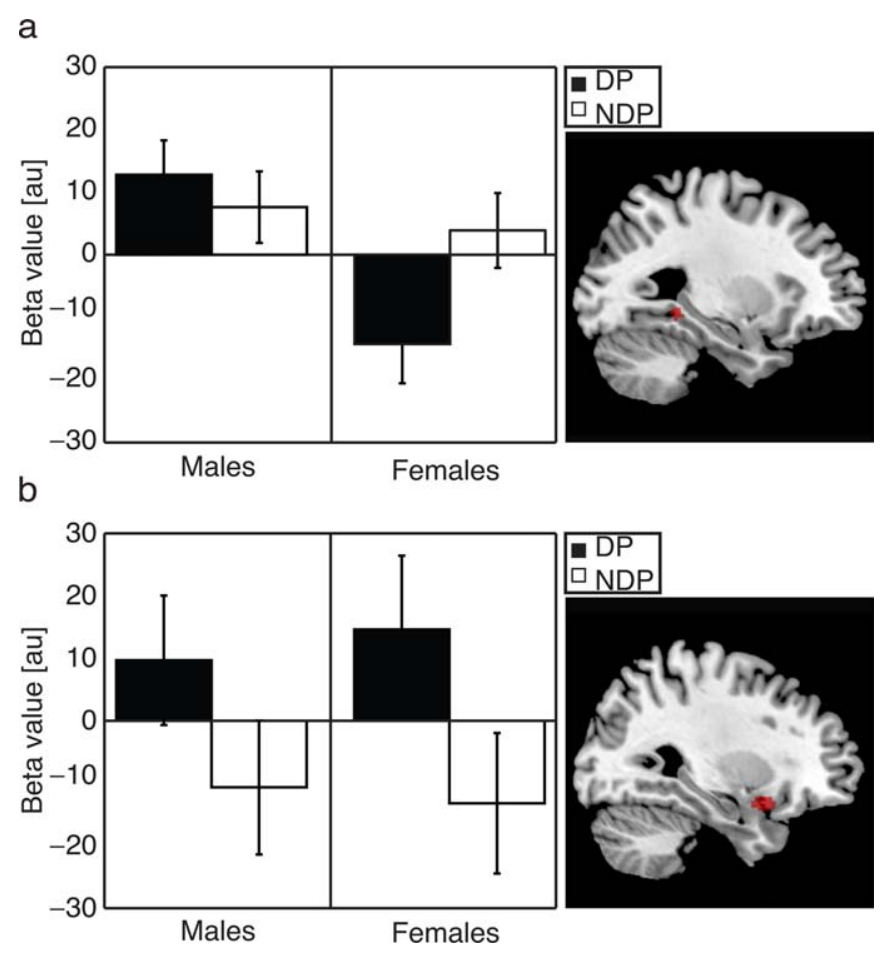

FIGURE 3. Memory representations differ between men and women 1 month following encoding. (a) Representation of DP > NDP 30 days after encoding in the PHG $(x=-28 y=-40$ $z=-6, P=0.014)$. Note that beta weights were extracted from the peak voxel. Weights were plotted for descriptive purposes only. (b) Representation of DP $>$ NDP 30 days after encoding in the inferior frontal gyrus $(x=28 y=12 z=-16, P=0.003)$. Note that beta weights were extracted from the peak voxel. Weights were plotted for descriptive purposes only. [Color figure can be viewed at wileyonlinelibrary.com] 
observed in females (Fig. 3a). This result was strengthened by a Time*Context*Sex interaction effect in the left PHG (Day 0Day $30^{*}$ Context ${ }^{*}$ Sex: $x=-32 \quad y=-42 \quad z=-4, \quad k=16$, $P_{\text {SVC }}=0.048$; Day $1-$ Day $30^{*}$ Context ${ }^{*}$ Sex: $x=-32 y=-42$ $\left.z=-4, k=8, P_{\text {SVC }}=0.077\right)$, indicating that the selective response to DP landmarks increased over time in males whereas the selective response to DP landmarks decreased in females (Fig. 2a,b). Similar effects were observed in the left cuneus $\left(x=-14 y=-66 z=24, k=368, P_{\mathrm{FWE}}<0.001\right)$, the right insula $\left(x=34 y=8 z=12, k=281, P_{\mathrm{FWE}}=.002\right)$ and in the left superior temporal lobe $(x=-38 y=-42 z=8, k=355$, $\left.P_{\text {FWE }}<0.001\right)$. No such interaction effects were observed in the right IFG, suggesting that male and female participants recruited this region equally (Fig 3b).

\section{DISCUSSION}

In this fMRI study, we systematically examined the effects of time and spatial context on the neural representation of landmark recognition memory. The data reinforced the idea that the hippocampus and posterior PHG support memory for landmarks. Though they played an equally vital role, the hippocampus and the posterior PHG subserved different functions. Whereas the hippocampus was initially involved in memory for all landmarks, the posterior PHG specifically responded to landmarks associated with a spatial context relevant for navigation. With time, functional reorganization occurred such that the representation of landmarks and the associated context developed in the IFG. The data further provide preliminary evidence for disparate consolidation trajectories in the male and the female brain. While the contribution of the posterior PHG was time-limited in females, no such pattern was observed in males. As a consequence, females depended upon the IFG when retrieving remote memory for landmarks and the associated context, while males relied both on the PHG and IFG.

The involvement of the hippocampus and posterior PHG in acquiring spatial memories is beyond dispute. Previous fMRI studies provided convergent evidence suggesting that the PHG mediates contextual associations such as binding an object to a spatial location or context (Eichenbaum and Cohen, 2001; Diana et al., 2007; Aminoff et al., 2013; Baumann and Mattingley, 2016). Specifically, landmarks associated with a relevant spatial context have been demonstrated to engage the PHG during encoding and subsequent retrieval (Janzen and van Turennout, 2004; Janzen et al., 2007, 2008; Janzen and Jansen, 2010; Schinazi and Epstein, 2010; Wegman and Janzen, 2011). In support of this, the current findings showed a selective response to relevant landmarks in the PHG $24 \mathrm{~h}$ following encoding, indicating that the PHG mediates object-location associations that are particularly relevant for navigation. The hippocampus, as evidenced by the results of this study, is initially implicated in the retrieval of all landmarks. The hippocampus may be characterized as an index or pointer to information registered in neocortical regions (e.g., posterior PHG) (Teyler and DiScenna, 1986). As such, the hippocampus could select contextual representations that make for a vivid recollection of a previously learned environment. However, the exact interplay between hippocampus and posterior PHG should be examined by future research.

In addition to areas associated with tasks requiring spatial memory processing, we observed increased activity in the prefrontal cortex with the passage of time. The most prominent increase was found in the right IFG, including Brodmann's areas 44 and 45 . These results are in agreement with those obtained by Takashima et al. (2009). They tested recent and remote memory for face-location associations and revealed increases with consolidation in neocortical areas including the bilateral IFG. There is abundant research showing that the IFG is involved in semantic processing (Cabeza and Nyberg, 2000; Thompson-Schill, 2003; Takashima et al., 2009, in press). This raises the possibility that remote memories for landmarks and their associated spatial context have a strong verbal component. For example, landmarks positioned at intersections may have been associated with a right or left turn. Indeed, neuroimaging studies provided evidence that semantic knowledge of objects and their associated actions activates the IFG (Martin et al., 1995; Ebisch et al., 2007). Yet, activity in the IFG was observed predominantly in the right hemisphere. Since language function is largely attributed to the left hemisphere, critics may question the legitimacy of a verbal interpretation of right IFG functioning. However, Binder et al. (2009) performed a meta-analysis of 120 functional neuroimaging studies and reported that $32 \%$ of the published foci involved in language processing were in the right hemisphere and only $68 \%$ in the left hemisphere. Specifically, recently published studies using repetitive transcranial magnetic stimulation showed that the right IFG is involved in language processing (Thiel et al., 2005; Naeser et al., 2011; Sollmann et al., 2014). Together, these results imply that activity in the right IFG reflects verbally mediated memory for landmarks and the associated spatial context. The exact nature of memory representations, however, remains to be determined.

Differential recruitment of the hippocampus, posterior PHG and IFG in recent and remote memory for landmarks may suggest a functional shift with consolidation from medial temporal lobe structures to a structure in the prefrontal cortex. A possible, albeit speculative, explanation is that memory representations of landmarks and spatial context reside in neocortical areas (including the posterior PHG) but converge in the hippocampus. With time, reorganization takes place such that the IFG mediates the retrieval of remote memory for landmarks and the associated context.

The results of this study do not easily reconcile with classical theories of memory consolidation. The standard consolidation theory postulates a role for neocortical areas in remote memory (McClelland et al., 1995; Squire and Alvarez, 1995; Frankland and Bontempi, 2005). Contrary to the observed findings, it implies that the PHG is capable of sustaining a permanent 
memory trace. It is not uncommon, however, for defenders of the standard theory of consolidation to study temporally limited memory in the PHG. For example, Teng and Squire (1999) tested patient E.P. who suffered from extensive bilateral damage to the hippocampus and the PHG. Whereas E.P. was severely impaired on tasks requiring spatial learning, he exhibited knowledge of spatial environments learned long ago. The authors concluded that spatial memories may diminish with time in the hippocampus and the PHG. In line with this finding, a meta-analysis of $66 \mathrm{fMRI}$ studies showed that recently learned environments require activation in the posterior PHG, whereas remotely learned environments are processed by a fronto-temporal parietal network (Boccia et al., 2014). The research outlined above and the results of this study suggest that neither the hippocampus nor the PHG is a permanent repository for spatial memories.

Opponents of the standard consolidation theory might argue that the functional reorganization with time is accompanied by a qualitative change in the nature of the memory representation. The idea that memories change with consolidation is in line with alternative theories like MTT, the BIC model, the scene construction theory and the transformation hypothesis (Nadel and Moscovitch, 1997; Eichenbaum et al., 2007; Hassabis and Maguire, 2007; Winocur et al., 2010). Memories registered in the hippocampus and posterior PHG may be characterized as contextually rich, whereas memories in the IFG might retain some of the essential features but few of the contextual details. Specifically, the process of semanticization potentially comprises a change in nature from spatial to verbal, where spatial representations are assumed to be contextually rich and verbal representations semanticized. Indeed, evidence confirms that both spatial memory and verbal memory are critically involved in the encoding of spatial information (Garden et al., 2002; Meilinger et al., 2008). It should be noted, however, that activity in the IFG was increased for landmarks associated with a relevant spatial context compared with an irrelevant spatial context, suggesting that some form of contextual memory was preserved.

The idea of a functional shift from the hippocampus and the posterior PHG to the IFG does not fully explain the observed findings of this study. While memory for landmarks associated with a relevant context initially appeared to be dependent on the PHG in both males and females, retrievalrelated activity patterns started to diverge. In females the response to landmarks associated with a relevant spatial context diminished in the PHG and strengthened in the IFG. In males, on the other hand, memories for landmarks associated with a relevant spatial context engaged the PHG regardless of the age of the memory.

Sex differences in spatial cognition are well established. Behavioral studies indicate that males outperform females on a variety of tests of spatial ability, such as keeping track of one's starting position (Hegarty et al., 2006; Persson et al., 2013) or finding one's way in a large-scale virtual environment (Moffat et al., 1998). The male advantage may be explained by differences in cognitive strategy. Males preferentially use a spatial strategy where they build upon a mental representation of the spatial layout of the environment (Lawton, 1994; Coluccia and Louse, 2004). On the other hand, females show a bias toward a verbal strategy, where contextual information such as a leftand right-turn is associated with a landmark (Lawton, 1994; Maguire et al., 1999; Coluccia and Louse, 2004). The premise that differences in navigational ability can be explained by the type of representation maintained in memory (i.e., spatial or verbal) is supported by work of Baumann et al. (2011). They demonstrated that good navigators rely on spatial information whereas bad navigators rely on both spatial and verbal information. In the same vein, the male advantage on tests of spatial ability is reduced when a verbal representation of landmarks can be used (Sandstrom et al., 1998).

Some studies suggest that behavioral differences between males and females parallel functional differences in the brain. For example, Grön et al. (2000) showed that when navigating through a virtual environment male participants engage the left hippocampus, whereas female participants recruit the right parietal and right prefrontal cortex. In accord with findings from Janzen and van Turennout (2004) and Janzen et al. (2007), however, the results of this study revealed neither behavioral nor functional sex differences during the first $24 \mathrm{~h}$ following encoding. Rather, differences between sexes became more apparent when memories aged. A possible explanation is that sex differences in remote memory for landmarks originate as a consequence of distinct forgetting rates of spatial and verbal memory components. In a behavioral study, Talamini and Gorree (2012) investigated decay of various memory components, including memory for object identity, which contained a strong verbal component, and memory for the associated temporal-spatial context, which contained a spatial component. Results showed stronger forgetting for contextual memories as compared with memory for object identity. This resonates with the observation that hippocampal and parahippocampal memory representations may degrade relatively fast (Takashima et al., 2006; Boccia et al., 2014). From this it could be inferred that males maintained access to the initially formed spatial memory in the posterior PHG, whereas females became more strongly dependent on a verbal representation of landmarks over time. Though the design of this study does not allow for firm conclusions, it approximates the predictions of alternative theories like MTT, BIC model, scene construction theory and transformation hypothesis. Importantly, the interpretation is supported by the finding that males showed a lower rate of forgetting for objects with a relevant spatial context than females did.

Although the involvement of the PHG in spatial processing is well established (Janzen and van Turennout, 2004; Janzen et al., 2007, 2008; Janzen and Jansen, 2010; Schinazi and Epstein, 2010; Wegman and Janzen, 2011), this study failed to demonstrate the involvement of the PHG in the representation of landmarks associated with a relevant context directly after encoding. This discrepancy could be attributed to a change in study design. Contrary to previous experiments, participants evaluated their level of confidence after they had made an old/ new judgment in response to a landmark. This might have 
provoked identity-related or semantic knowledge as opposed to location-related or spatial knowledge. Probing memory for object-identity rather than object-location could have overshadowed the item-context association that was at test here. Following overnight sleep however, the PHG did respond to landmarks associated with a navigationally relevant spatial context. It can be argued that the selective response of the PHG to landmarks associated with a relevant context has strengthened progressively with consolidation. This is in line with earlier studies reporting increases in hippocampal and parahippocampal function with time (Bosshardt et al., 2005; Rekkas and Constable, 2005). Of particular relevance to this study, Janzen and colleagues $(2007,2008)$ demonstrated that memory for relevant landmarks following a 24-h encoding-retrieval interval induced increased activity in bilateral PHG compared with recent memory for relevant landmarks.

This study was limited by its sample size and, as a consequence, the results must be treated with care. However, the results yield a clear objective for future research. It may prove informative to examine sex differences in a larger sample, especially since previous studies on the neurobiology of memory were similarly restricted by methodological constraints such as small sample size (Grön et al., 2000; Nyberg et al., 2000; Piefke et al., 2005; Sneider et al., 2011; Persson et al., 2013). Specifically, study designs that enable systematic examination of the nature of contextual memories (i.e., spatial or verbal) in males and females could be a valuable contribution to the discussion. It may inform us about the underlying mechanism of sex differences in spatial cognition and it potentially unifies the different accounts on memory consolidation.

In conclusion, the data reinforce the idea that the neural representation of landmarks changes with time. The response to landmarks diminished with time in the hippocampus and the PHG and strengthened in the IFG. Furthermore, we provide preliminary evidence of disparate neural changes over time in males and females. The findings suggest that differences in strategy use are amplified when memories age: while males may rely upon a spatial representation of landmarks and their locations regardless of the age of the memory, females might develop a more verbal representation of landmarks with the passage of time.

\section{REFERENCES}

Aguirre GK, Detre JA, Alsop DC, Desposito M. 1996. The parahippocampus subserves topographical learning in man. Cereb Cortex 6:823-829.

Aminoff EM, Kveraga K, Bar M. 2013. The role of the parahippocampal cortex in cognition. Trends Cognit Sci 17:379-390.

Andreano JM, Cahill L. 2009. Sex influences on the neurobiology of learning and memory. Learn Mem 16:248-266.

Baumann O, Chan E, Mattingley JB. 2010. Dissociable neural circuits for encoding and retrieval of object locations during active navigation in humans. NeuroImage 49:2816-2825.
Baumann O, Mattingley JB. 2016. Functional organization of the parahippocampal cortex: Dissociable roles for context representations and the perception of visual scenes. J Neurosci 36:2536-2542.

Baumann O, Skilleter AJ, Mattingley JB. 2011. Short-term memory maintenance of object locations during active navigation: Which working memory subsystem is essential? PLoS One 6:e19707.

Binder JR, Desai RH. 2011. The neurobiology of semantic memory. Trends Cognit Sci 15:527-536.

Binder JR, Desai RH, Graves WW, Conant LL. 2009. Where is the semantic system? A critical review and meta-analysis of 120 functional neuroimaging studies. Cereb Cortex 19:2767-2796.

Boccia M, Nemmi F, Guariglia C. 2014. Neuropsychology of environmental navigation in humans: Review and meta-analysis of fMRI studies in healthy participants. Neuropsychol Rev 24:236-251.

Bontempi B, Laurent-Demir C, Destrade C, Jaffard R. 1999. Timedependent reorganization of brain circuitry underlying long-term memory storage. Nature 400:671-675.

Bosshardt S, Schmidt CF, Jaermann T, Degonda N, Boesiger P, Nitsch RM, Hock C, Henke K. 2005. Effects of memory consolidation on human hippocampal activity during retrieval. Cortex 41:486-498.

Burgess N, Maguire EA, O'Keefe J. 2002. The human hippocampus and spatial and episodic memory. Neuron 35:625-641.

Burwell RD, Bucci DJ, Sanborn MR, Jutras MJ. 2004. Perirhinal and postrhinal contributions to remote memory for context. J Neurosci 24:11023-11028.

Cabeza R, Nyberg L. 2000. Imaging cognition II: An empirical review of 275 PET and fMRI studies. J Cogn Neurosci 12:1-47.

Coluccia E, Louse G. 2004. Gender differences in spatial orientation: A review. J Environ Psychol 24:329-340.

Diana RA, Yonelinas AP, Ranganath C. 2007. Imaging recollection and familiarity in the medial temporal lobe: A three-component model. Trends Cognit Sci 11:379-386.

Douville K, Woodard JL, Seidenberg M, Miller SK, Leveroni CL, Nielson KA, Franczak M, Antuono P, Rao SA. 2005. Medial temporal lobe activity for recognition of recent and remote famous names: An event-related fMRI study. Neuropsychologia 43:693703.

Duzel E, Habib R, Rotte M, Guderian S, Tulving E, Heinze HJ. 2003. Human hippocampal and parahippocampal activity during visual associative recognition memory for spatial and nonspatial stimulus configurations. J Neurosci 23:9439-9444.

Ebisch SJH, Babiloni C, Del Gratta C, Ferretti A, Perrucci MG, Caulo M, Sitskoorn MM, Romani GL. 2007. Human neural systems for conceptual knowledge of proper object use: A functional magnetic resonance imaging study. Cereb Cortex 17:2744-2751.

Eichenbaum H, Cohen N J. 2001. From Conditioning to Conscious Recollection: Memory Systems of the Brain. New York: Oxford University Press.

Eichenbaum H, Yonelinas AP, Ranganath C. 2007. The medial temporal lobe and recognition memory. Annu Rev Neurosci 30:123152.

Epstein R, Kanwisher N. 1998. A cortical representation of the local visual environment. Nature 392:598-601.

Euston DR, Gruber AJ, McNaughton BL. 2012. The role of medial prefrontal cortex in memory and decision making. Neuron 76 : 1057-1070.

Frankland PW, Bontempi B. 2005. The organization of recent and remote memories. Nat Rev Neurosci 6:119-130.

Garden S, Cornoldi C, Logie RH. 2002. Visuo-spatial working memory in navigation. Appl Cogn Psychol 16:35-50.

Goh JOS, Siong SC, Park D, Gutchess A, Hebrank A, Chee MWL. 2004. Cortical areas involved in object, background, and object background processing revealed with functional magnetic resonance adaptation. J Neurosci 24:10223-10228.

Grön G, Wunderlich AP, Spitzer M, Tomczak R, Riepe MW. 2000. Brain activation during human navigation: Gender-different neural networks as substrate of performance. Nat Neurosci 3:404-408. 
Haist F, Gore JB, Mao H. 2001. Consolidation of human memory over decades revealed by functional magnetic resonance imaging. Nat Neurosci 4:1139-1145.

Hassabis D, Chu C, Rees G, Weiskopf N, Molyneux PD, Maguire EA. 2009. Decoding neuronal ensembles in the human hippocampus. Curr Biol 19:546-554.

Hassabis D, Maguire EA. 2007. Deconstructing episodic memory with construction. Trends Cognit Sci 11:299-306.

Hayasaka S, Nichols TE. 2003. Validating cluster size inference: Random field and permutation methods. NeuroImage 20:2343-2356.

Hayes SM, Ryan L, Schnyer DM, Nadel L. 2004. An fMRI study of episodic memory: Retrieval of object, spatial, and temporal information. Behav Neurosci 118:885-896.

Hegarty M, Montello DR, Richardson AE, Ishikawa T, Lovelace K. 2006. Spatial abilities at different scales: Individual differences in aptitude-test performance and spatial-layout learning. Intelligence 34:151-176.

Herdman KA, Calarco N, Moscovitch M, Hirshhorn M, Rosenbaum RS. 2015. Impoverished descriptions of familiar routes in three cases of hippocampal/medial temporal lobe amnesia. Cortex 71: 248-263.

Hirshhorn M, Grady C, Rosenbaum RS, Winocur G, Moscovitch M. 2012. The hippocampus is involved in mental navigation for a recently learned, but not a highly familiar environment: A longitudinal fMRI study. Hippocampus 22:842-852.

Holden MP, Duff-Canning SJ, Hampson E. 2015. Sex differences in the weighting of metric and categorical information in spatial location memory. Psychol Res-Psychol Forsch 79:1-18.

Howard LR, Kumaran D, Olafsdottir HF, Spiers HJ. 2011. Double dissociation between hippocampal and parahippocampal responses to object-background context and scene novelty. J Neurosci 31: 5253-5261.

Janzen G, Jansen C. 2010. A neural wayfinding mechanism adjusts for ambiguous landmark information. NeuroImage 52:364-370.

Janzen G, Jansen C, van Turennout M. 2008. Memory consolidation of landmarks in good navigators. Hippocampus 18:40-47.

Janzen G, van Turennout M. 2004. Selective neural representation of objects relevant for navigation. Nat Neurosci 7:673-677.

Janzen G, Wagensveld B, van Turennout M. 2007. Neural representation of navigational relevance is rapidly induced and long lasting. Cereb Cortex 17:975-981.

Kim JJ, Fanselow MS. 1992. Modality-specific retrograde amnesia of fear. Science 256:675-677.

Kolb B, Cioe J. 1996. Sex-related differences in cortical function after medial frontal lesions in rats. Behav Neurosci 110:1271-1281.

Lawton CA. 1994. Gender differences in way-finding strategies: Relationship to spatial ability and spatial anxiety. Sex Roles 30:765779.

Linsley D, MacEvoy SP. 2015. Encoding-stage crosstalk between object- and spatial property-based scene processing pathways. Cereb Cortex 25:2267-2281.

Maguire EA, Burgess N, O’Keefe J. 1999. Human spatial navigation: Cognitive maps, sexual dimorphism, and neural substrates. Curr Opin Neurobiol 9:171-177.

Maguire EA, Frith CD, Burgess N, Donnett JG, O'Keefe J. 1998. Knowing where things are: Parahippocampal involvement in encoding object locations in virtual large-scale space. J Cogn Neurosci 10:61-76.

Maldjian JA, Laurienti PJ, Kraft RA, Burdette JH. 2003. An automated method for neuroanatomic and cytoarchitectonic atlas-based interrogation of fMRI data sets. NeuroImage 19:1233-1239.

Martin A, Haxby JV, Lalonde FM, Wiggs CL, Ungerleider LG. 1995. Discrete cortical regions associated with knowledge of color and knowledge of action. Science 270:102-105.

Maviel T, Durkin TP, Menzaghi F, Bontempi B. 2004. Sites of neocortical reorganization critical for remote spatial memory. Science 305:96-99.
McClelland JL, McNaughton BL, Oreilly RC. 1995. Why there are complementary learning-systems in the hippocampus and neocortex: Insights from the successes and failures of connectionist models of learning and memory. Psychol Rev 102:419-457.

Meilinger T, Knauff M, Bulthoff HH. 2008. Working memory in wayfinding - A dual task experiment in a virtual city. Cogn Sci 32:755-770.

Moffat SD, Hampson E, Hatzipantelis M. 1998. Navigation in a "virtual" maze: Sex differences and correlation with psychometric measures of spatial ability in humans. Evol Hum Behav 19: 73-87.

Montello DR, Lovelace KL, Golledge RG, Self CM. 1999. Sex-related differences and similarities in geographic and environmental spatial abilities. Ann Assoc Am Geogr 89:515-534.

Moscovitch M, Rosenbaum RS, Gilboa A, Addis DR, Westmacott R, Grady C, McAndrews MP, Levine B, Black S, Winocur G, Nadel L. 2005. Functional neuroanatomy of remote episodic, semantic and spatial memory: A unified account based on multiple trace theory. J Anat 207:35-66.

Nadel L, Moscovitch M. 1997. Memory consolidation, retrograde amnesia and the hippocampal complex. Curr Opin Neurobiol 7: 217-227.

Naeser MA, Martin PI, Theoret H, Kobayashi M, Fregni F, Nicholas M, Tormos JM, Steven MS, Baker EH, Pascual-Leone A. 2011. TMS suppression of right pars triangularis, but not pars opercularis, improves naming in aphasia. Brain Lang 119: 206-213.

Nieuwenhuis ILC, Takashima A. 2011. The role of the ventromedial prefrontal cortex in memory consolidation. Behav Brain Res 218: 325-334.

Nyberg L, Habib R, Herlitz A. 2000. Brain activation during episodic memory retrieval: Sex differences. Acta Psychol (Amst) 105:181194.

O’Keefe J, Nadel L. 1978. The Hippocampus As a Cognitive Map. Oxford: Oxford University Press.

Persson J, Herlitz A, Engman J, Morell A, Sjolie D, Wikstrom J, Soderlund H. 2013. Remembering our origin: Gender differences in spatial memory are reflected in gender differences in hippocampal lateralization. Behav Brain Res 256:219-228.

Piefke M, Weiss PH, Markowitsch HJ, Fink GR. 2005. Gender differences in the functional neuroanatomy of emotional episodic autobiographical memory. Hum Brain Mapp 24:313-324.

Poser BA, Versluis MJ, Hoogduin JM, Norris DG. 2006. BOLD contrast sensitivity enhancement and artifact reduction with multiecho EPI: Parallel-acquired inhomogeneity-desensitized fMRI. Magn Reson Med 55:1227-1235.

Ranganath C, Ritchey M. 2012. Two cortical systems for memoryguided behaviour. Nat Rev Neurosci 13:713-726.

Rekkas PV, Constable RT. 2005. Evidence that autobiographic memory retrieval does not become independent of the hippocampus: An fMRI study contrasting very recent with remote events. J Cogn Neurosci 17:1950-1961.

Remondes M, Schuman EM. 2004. Role for a cortical input to hippocampal area CA1 in the consolidation of a long-term memory. Nature 431:699-703.

Roof RL, Zhang Q, Glasier MM, Stein DG. 1993. Gender-specific impairment on Morris water maze task after entorhinal cortex lesions. Behav Brain Res 57:47-51.

Rosenbaum RS, Priselac S, Kohler S, Black SE, Gao FQ, Nadel L, Moscovitch M. 2000. Remote spatial memory in an amnesic person with extensive bilateral hippocampal lesions. Nat Neurosci 3: 1044-1048.

Rosenbaum RS, Ziegler M, Winocur G, Grady CL, Moscovitch M. 2004. "I have often walked down this street before": fMRI studies on the hippocampus and other structures during mental navigation of an old environment. Hippocampus 14:826-835. 
Sandstrom NJ, Kaufman J, Huettel SA. 1998. Males and females use different distal cues in a virtual environment navigation task. Cogn Brain Res 6:351-360.

Saucier DM, Green SM, Leason J, MacFadden A, Bell S, Elias LJ. 2002. Are sex differences in navigation caused by sexually dimorphic strategies or by differences in the ability to use the strategies? Behav Neurosci 116:403-410.

Schinazi VR, Epstein RA. 2010. Neural correlates of real-world route learning. NeuroImage 53:725-735.

Shelton AL, Gabrieli JDE. 2002. Neural correlates of encoding space from route and survey perspectives. J Neurosci 22:2711-2717.

Smith CN, Squire LR. 2009. Medial Temporal Lobe Activity during retrieval of semantic memory is related to the age of the memory. J Neurosci 29:930-938.

Sneider JT, Sava S, Rogowska J, Yurgelun-Todd DA. 2011. A preliminary study of sex differences in brain activation during a spatial navigation task in healthy adults. Percept Mot Skills 113: 461-480.

Sollmann N, Tanigawa N, Ringel F, Zimmer C, Meyer B, Krieg SM. 2014. Language and its right-hemispheric distribution in healthy brains: An investigation by repetitive transcranial magnetic stimulation. NeuroImage 102:776-788.

Sommer T, Rose M, Weiller C, Buchel C. 2005. Contributions of occipital, parietal and parahippocampal cortex to encoding of object-location associations. Neuropsychologia 43:732-743.

Spiers HJ, Maguire EA. 2007. The neuroscience of remote spatial memory: A tale of two cities. Neuroscience 149:7-27.

Squire LR, Alvarez P. 1995. Retrograde amnesia and memory consolidation: A neurobiological perspective. Curr Opin Neurobiol 5: 169-177.

Squire LR, Genzel L, Wixted JT, Morris RG. 2015. Memory consolidation. Cold Spring Harb Perspect Biol 7:a021766.

Takashima A, Bakker I, van Hell JG, Janzen G, McQueen JM. Interaction between episodic and semantic memory networks in the acquisition and consolidation of novel spoken words. Brain Lang (in press).

Takashima A, Nieuwenhuis ILC, Jensen O, Talamini LM, Rijpkema M, Fernandez G. 2009. Shift from hippocampal to neocortical centered retrieval network with consolidation. J Neurosci 29: 10087-10093.
Takashima A, Petersson KM, Rutters F, Tendolkar I, Jensen O, Zwarts MJ, McNaughton BL, Fernandez G. 2006. Declarative memory consolidation in humans: A prospective functional magnetic resonance imaging study. Proc Natl Acad Sci USA 103: 756-761.

Talamini LM, Gorree E. 2012. Aging memories: Differential decay of episodic memory components. Learn Mem 19:239-246.

Teng E, Squire LR. 1999. Memory for places learned long ago is intact after hippocampal damage. Nature 400:675-677.

Teyler TJ, DiScenna P. 1986. The hippocampal memory indexing theory. Behav Neurosci 100:147-154.

Thiel A, Habedank B, Winhuisen L, Herholz K, Kessler J, Haupt WF, Heiss WD. 2005. Essential language function of the right hemisphere in brain tumor patients. Ann Neurol 57:128-131.

Thompson-Schill SL. 2003. Neuroimaging studies of semantic memory: Inferring "how" from "where". Neuropsychologia 41:280-292.

Wegman J, Fonteijn HM, van Ekert J, Tyborowska A, Jansen C, Janzen G. 2014. Gray and white matter correlates of navigational ability in humans. Hum Brain Mapp 35:2561-2572.

Wegman J, Janzen G. 2011. Neural encoding of objects relevant for navigation and resting state correlations with navigational ability. J Cogn Neurosci 23:3841-3854.

Wiltgen BJ, Brown RAM, Talton LE, Silva AJ. 2004. New circuits for old memories: The role of the neocortex in consolidation. Neuron 44:101-108.

Winocur G. 1990. Anterograde and retrograde amnesia in rats with dorsal hippocampal or dorsomedial thalamic lesions. Behav Brain Res 38:145-154.

Winocur G, Moscovitch M, Bontempi B. 2010. Memory formation and long-term retention in humans and animals: Convergence towards a transformation account of hippocampal-neocortical interactions. Neuropsychologia 48:2339-2356.

Yamashita K, Hirose S, Kunimatsu A, Aoki S, Chikazoe J, Jimura K, Masutani Y, Abe O, Ohtomo K, Miyashita Y, Konishi S. 2009. Formation of long-term memory representation in human temporal cortex related to pictorial paired associates. J Neurosci 29: $10335-10340$.

Zola-Morgan SM, Squire LR. 1990. The primate hippocampal formation: Evidence for a time-limited role in memory storage. Science 250:288-290. 